\title{
Sensorless Vector Controller for a Synchronous Reluctance Motor
}

\author{
Milutin G. Jovanović, Robert E. Betz, Member, IEEE, and Don Platt, Member, IEEE
}

\begin{abstract}
A new high-performance sensorless speed vector controller that implements the maximum torque per ampere control strategy for the inverter-driven synchronous reluctance machine is presented in this paper. It is based on a parameterdependent technique for on-line estimation of rotor position and angular velocity at the control rate. The current ripple principle is used to estimate position. The estimates are fed to a conventional closed-loop observer to predict the new position and angular velocity. The very high accuracy of the sensorless control algorithm at both low and high speeds is confirmed by experimental results.
\end{abstract}

Index Terms - Angular velocity observer, sensorless control, synchronous reluctance machines.

\section{INTRODUCTION}

$\mathbf{T}$ HE high-performance rotor-oriented vector control of the cageless inverter-fed synchronous reluctance machine (Syncrel) requires an accurate knowledge of rotor position to convert the measurable stator quantities into their rotating frame equivalents. The position information is traditionally provided by measurements using costly transducers, such as optical encoders or magnetic resolvers. In order to make the Syncrel drive less expensive and more robust compared to its induction machine counterpart, recent work has focused on investigating various position estimation techniques that would allow the removal of shaft position sensors [1]-[7]. An additional motivation for the renewed interest in Syncrel is undoubtedly its inherent saliency and, thus, amenability to sensorless operation.

Although the sensorless Syncrel drive is of lower cost and more mechanically robust, its control performance is usually compromised, and controller design is generally more complicated. The literature consistently shows that it is not easy to implement sensorless control in real time, and only some authors have succeeded in achieving this [1], [2], [6].

Paper IPCSD 97-66, presented at the 1996 Industry Applications Society Annual Meeting, San Diego, CA, October 6-10, and approved for publication in the IEEE TRANSACTIONS ON INDUSTRY APPLICATIONS by the Industrial Drives Committee of the IEEE Industry Applications Society. Manuscript released for publication October 20, 1997.

M. G. Jovanović was with the Department of Electrical and Computer Engineering, University of Newcastle, Callaghan, NSW 2308, Australia. He is now with the School of Electrical Engineering, Electronics and Physics, Liverpool John Moores University, Liverpool L3 3AF, U.K.

R. E. Betz is with the Institute of Energy Technology, Department of Electrical Energy Conservation, Aalborg University, DK-9220 Aalborg East, Denmark, on leave from the Department of Electrical and Computer Engineering, University of Newcastle, Callaghan, NSW 2308, Australia.

D. Platt is with the Department of Electrical and Computer Engineering, University of Wollongong, Wollongong, NSW 2522, Australia.

Publisher Item Identifier S 0093-9994(98)02563-8.
The others carried out detailed studies of different estimation algorithms and, while significant from a theoretical viewpoint, the results are preliminary and with no experimental support [4], [5], [7].

The basic principle used by most techniques [1], [3]-[6] is the variation of stator inductances with rotor position, which allows position estimation down to zero speed. This variation is enhanced with larger saliency ratios and can be detected in the switching ripples on the current waveforms [4]-[6] or by the magnetic coupling coefficients between windings [3]. These two approaches are combined in [1] for both low and high speeds, respectively. A Kalman filter is then used to obtain the optimal position and velocity estimates. An effective flux-oriented speed controller based on the torque vector control principle [7] is described in [2]. Unlike the previous techniques, it does not require a rotor position information at all, but only needs to know the flux position. Reference [8] outlines the versatile sensorless scheme potentially applicable to all salient ac machines.

The main contribution of the work presented in [2], [6], and [8] is the development of viable position estimation techniques independent of machine parameters and operating point. The advantage of the control algorithm in [1] is that it makes it possible to obtain satisfactory control of shaft speed over the whole range, in contrast to those in [6] and [2], the applications of which are limited to low and high speeds, respectively.

The limitations of the existing control algorithms are as follows: 1) relatively modest position estimation accuracy of approximately $10^{\circ}$ electrical [1], [6]; 2) low estimate update rate and, hence, poor control performance [3]; 3) the use of special switching procedures to force the inverter into a desired diagnostic state, in order to carry out measurements relevant for estimation [1], [3]; and 4) an injection of special high-frequency signals which have to be filtered to obtain the position estimate [8].

This paper is, in some sense, an extension of the work initiated in [4]. It presents a real-time software implementation of a new observer-based sensorless algorithm for the maximum torque per ampere control strategy that overcomes some of the above deficiencies. It includes a parameter-dependent technique for estimating the rotor position on-line from the measurements of two stator currents and the inverter dc-link voltage. The position is estimated using the saturated $d-q$ model equations in discrete form and linear approximation of the rate of change of current in the switching ripples. This makes the control algorithm applicable throughout the entire speed range and under all loading conditions of the machine. 
A startup procedure is required to establish the initial position of the rotor $d$ axis, after which the algorithm can be run. The excellent performance of the controller is experimentally verified on a 5.8-kW axially laminated Syncrel.

\section{Position Estimation TeChNiQue}

The fundamental principle of the estimation technique is the detection of stator winding inductance variations with rotor position, by examination of the switching ripple on the current waveforms. It actually represents an improved version of the estimation algorithm presented by the same authors in [4], which is similar to that in [5]. A significant improvement is that the saturation effects are included.

Consider conventional Park's $d q$ model equations for the Syncrel using standard notation:

$$
\begin{aligned}
e_{d} & =u_{d}-R i_{d}=\frac{d \lambda_{d}}{d t}-\omega \lambda_{q} \\
e_{q} & =u_{q}-R i_{q}=\frac{d \lambda_{q}}{d t}+\omega \lambda_{d} \\
\tau & =\frac{q}{2} p\left[L_{d}\left(i_{d}\right)-L_{q}\right] i_{d} i_{q} \\
J \frac{d \omega}{d t} & =p\left(\tau-\tau_{\text {load }}\right)-f \omega \\
\omega & =\frac{d \theta}{d t} .
\end{aligned}
$$

Developing the voltage equations in terms of $d q$ currents and taking into account the saturation in the high-permeance rotor axis ( $d$ axis), one obtains

$$
\begin{aligned}
e_{d} & =\left[L_{d}\left(i_{d}\right)+i_{d} L_{d}^{\prime}\left(i_{d}\right)\right] \frac{d i_{d}}{d t}-\omega L_{q} i_{q} \\
& =\tilde{L}_{d} \frac{d i_{d}}{d t}-\omega L_{q} i_{q} \\
e_{q} & =L_{q} \frac{d i_{q}}{d t}+\omega L_{d}\left(i_{d}\right) i_{d}
\end{aligned}
$$

where $e$ is the induced voltage and $L_{d}^{\prime}=d L_{d} / d i_{d}$.

The value of stator resistance $R$ as measured by a simple dc test is somewhat increased to compensate for skin effects and temperature variations. The measurements for the saturation characteristic, $L_{d}$ versus $i_{d}$ and $L_{d}^{\prime}$ versus $i_{d}$, are stored in a lookup table, the values of which have been determined by off-line testing (see the Appendix). The $q$ axis is dominated by air, therefore, $L_{q}$ is assumed to be constant. All relevant machine parameters are summarized in the Appendix.

In order to solve (2) for the rotor electrical angular position $\theta$, it is necessary to relate quantities in the rotor $d q$ frame directly to their measurable counterparts in the $d_{s} q_{s}$ stator frame of reference. This can be achieved by applying the following transformation equation, which can be derived from the vector diagram in Fig. 1:

$$
\bar{X}=X_{d_{s}}+j X_{q_{s}}=\left(X_{d}+j X_{q}\right) e^{j \theta}
$$

where $X$ represents either the induced voltage $e$, current $i$, or terminal voltage $u$.

Consider a Syncrel being fed by a space-vector-based pulsewidth modulation (PWM) inverter [9]. Depending on the switching status of the legs, the inverter can produce six

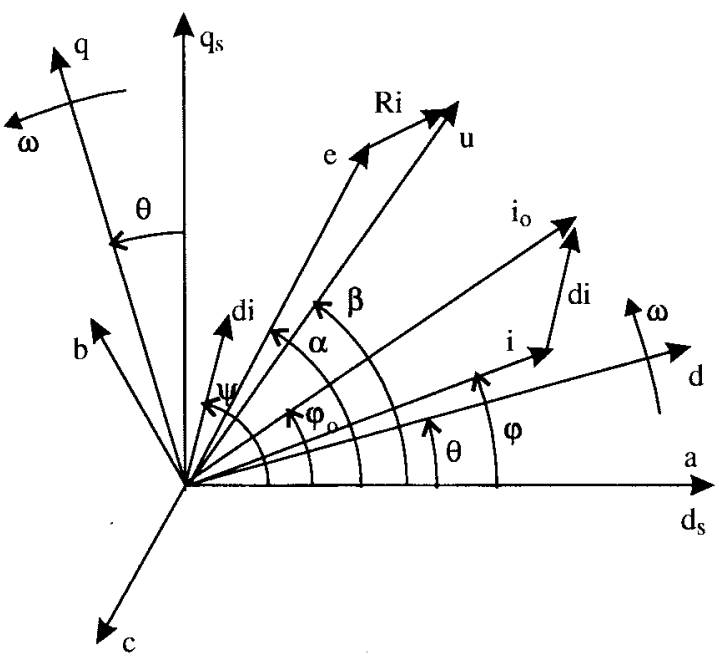

Fig. 1. Phasor diagram.

nonzero- and two zero-voltage phasors. The nonzero phasors are displaced by $\pi / 3$ radians

$$
\bar{u}=u_{d_{s}}+j u_{q_{s}}=\frac{2}{3} V_{\mathrm{dc}} e^{j k \pi / 3}, \quad k=0,1, \cdots, 5
$$

where $V_{\mathrm{dc}}$ is a dc-link voltage magnitude.

Assuming no neutral connection, the $d_{s} q_{s}$ currents of the machine can be determined from the measured instantaneous values of two phase currents (e.g., $i_{a_{m}}$ and $i_{b_{m}}$ ) using an expression

$$
\begin{aligned}
\bar{i} & =i_{d_{s}}+j i_{q_{s}}=\frac{2}{3}\left(i_{a_{m}}+i_{b_{m}} e^{j 2 \pi / 3}+i_{c_{m}} e^{-j 2 \pi / 3}\right) \\
& =i_{a_{m}}+j \frac{i_{a_{m}}+2 i_{b_{m}}}{\sqrt{3}} .
\end{aligned}
$$

Differentiating further (3) with $X \triangleq i$, one gets

$$
\begin{aligned}
d i_{d}+j d i_{q} & =d \bar{i} e^{-j \theta}-j \bar{i} e^{-j \theta} d \theta \\
d i_{d_{s}}+j d i_{q_{s}} & =d \bar{i}=\bar{i}_{o}-\bar{i}=i_{o_{d_{s}}}-i_{d_{s}}+j\left(i_{o_{q_{s}}}-i_{q_{s}}\right)
\end{aligned}
$$

where $d \bar{i}$ is the phasor for the incremental current, and $\bar{i}$ and $\bar{i}_{o}$ are the phasors that correspond to the current samples at the beginning (after switching) and the end (before next switching) of a particular switching interval, respectively. The parameter $d t$ in (2) is the time increment between the sampling instants. The number of sampling intervals contained in $d t$ can be accurately determined from a knowledge of the sampling rate and the inverter switching times which are implicitly available, as the PWM waveform is generated by software. A simple algorithm is implemented to achieve this.

Note that the first and second terms on the right-hand side of the first equation of (6) respectively account for change in current phasor and movement of the $d q$ frame over $d t$. The linear approximation of the current derivative used in (6) gives reasonable accuracy in PWM drives, as $d t$ is usually very small in relation to the $L R$ time constant of the machine. An inspection of the experimental current ripple waveforms confirms this assumption, since they are virtually triangular in shape. 
After substituting $d \bar{i}=d i e^{j \psi}$ and $\bar{i}=i e^{j \varphi}$ into (6), the rotor frame components of $\overline{d i}$ become

$$
\begin{aligned}
& d i_{d}=d i \cos (\psi-\theta)+\omega i \sin (\varphi-\theta) d t \\
& d i_{q}=d i \sin (\psi-\theta)-\omega i \cos (\varphi-\theta) d t
\end{aligned}
$$

where $\varphi$ and $\psi$ are defined in Fig. 1. From the same figure, the following relations are also obvious:

$$
\begin{aligned}
& \bar{e}=e_{d}+j e_{q}=e \cos (\alpha-\theta)+j e \sin (\alpha-\theta) \\
& \bar{i}=i_{d}+j i_{q}=i \cos (\varphi-\theta)+j i \sin (\varphi-\theta) .
\end{aligned}
$$

The initial induced voltage equations (2), together with (8), can be now rearranged to the form

$$
\begin{aligned}
& e \cos (\alpha-\theta) d t \\
& \quad=\tilde{L}_{d} d i \cos (\psi-\theta)+\left(\tilde{L}_{d}-L_{q}\right) \omega i \sin (\varphi-\theta) d t \\
& \quad e \sin (\alpha-\theta) d t \\
& \quad=L_{q} d i \sin (\psi-\theta)+\left(L_{d}-L_{q}\right) \omega i \cos (\varphi-\theta) d t .
\end{aligned}
$$

These equations can be solved for $\theta$

$$
\begin{aligned}
& \theta_{1}=\tan ^{-1} \frac{B+\sqrt{B^{2}-4 A C}}{2 A} \\
& \theta_{2}=\tan ^{-1} \frac{B-\sqrt{B^{2}-4 A C}}{2 A} \\
& \theta_{3}=\theta_{1}+\pi \quad \theta_{4}=\theta_{2}+\pi
\end{aligned}
$$

where

$$
\begin{aligned}
A= & \left(L_{d}-L_{q}\right)\left(\tilde{L}_{d} d i_{q_{s}}-e_{q_{s}} d t\right) i_{q_{s}} \\
& +\left(\tilde{L}_{d}-L_{q}\right)\left(e_{d_{s}} d t-L_{q} d i_{d_{s}}\right) i_{d_{s}} \\
B= & \left(\tilde{L}_{d}+L_{d}-2 L_{q}\right)\left(e_{q_{s}} i_{d_{s}}+e_{d_{s}} i_{q_{s}}\right) d t \\
& -\left(\tilde{L}_{d} L_{d}-L_{q}^{2}\right)\left(i_{d_{s}} d i_{q_{s}}+i_{q_{s}} d i_{d_{s}}\right) \\
C= & \left(L_{d}-L_{q}\right)\left(\tilde{L}_{d} d i_{d_{s}}-e_{d_{s}} d t\right) i_{d_{s}} \\
& +\left(\tilde{L}_{d}-L_{q}\right)\left(e_{q_{s}} d t-L_{q} d i_{q_{s}}\right) i_{q_{s}} \\
e_{d_{s}, q_{s}}= & u_{d_{s}, q_{s}}-R i_{d_{s}, q_{s}} .
\end{aligned}
$$

The terminal voltages and currents in the stationary $d_{s} q_{s}$ frame (Fig. 1) used in the previous expressions are determined by (4) and (5), respectively. The corresponding incremental currents are defined in (6).

It is also possible to solve for $\omega$ using (9), however, the resultant expression is more sensitive to numerical errors, noise effects, and other real-time implementation inaccuracies. The value derived from this solution would require additional filtering and computation, which would, in turn, compromise the control performance. These reasons contributed to the abandonment of this approach.

The main advantage of the proposed estimation technique is that it provides one estimate per leg switched. Therefore, over one control interval, there is generally a number of solutions of (9). Considering that the PWM algorithm being implemented has double-edged modulation [9], there are usually four estimates available, as four legs are normally switched per control interval.
Such a high estimation rate has two important implications. Firstly, further processing is possible to help eliminate erroneous results and obtain the most accurate value to be used in the control procedure. This is achieved with a standard angular velocity observer [10], which allows accurate estimates of both $\omega$ and $\theta$ to be deduced from the noisy estimates $\theta_{\text {est }}$ coming directly from the solution of (10). Note that the use of the observer introduces another layer of parameter dependence, as the load parameters need to be known.

Secondly, the technique is applicable at any speed, including standstill, and under all loading conditions of the machine. An estimate is less sensitive to noise and measurement quantization and, hence, more accurate for larger current ripples. At low speeds, the current ripple is low, as there is no back EMF to decrease the currents when the zero vector is applied. In addition, when voltage is being applied from the supply, the pulses are narrow. On the other hand, at higher speeds, the accuracy is improved due to the back EMF induced ripple. At either speed, however, the estimation errors are smaller for the inverter leg switching states when most of the driving voltage (applied or induced) is in the low permeance $q$ axis, as the rate of change of current is higher.

For the best performance of the position estimator, the current sampling rate should be sufficiently high, so that the extremities of a switching cycle can be accurately determined. A sufficient rate is clearly dependent on the switching rate of the inverter; for high switching rates, the current sampling system should sample faster as a particular switching voltage is being held for a shorted time. In addition, high sampling rates allow more accurate determination of the current values at the beginning and end of a switching cycle. This is important in order to maximize the measured current ripple and becomes increasingly important at lower speeds.

The existing A/D sampling system (see Section V-A) with the maximum sampling rate of $130 \mathrm{kHz}$ is not the best solution in this regard. In addition, it is a multiplexed board $\left(i_{a_{m}}\right.$ is sampled first, then $i_{b_{m}}$, and, finally, $\left.V_{\mathrm{dc}_{\mathrm{m}}}\right)$. This reduces the effective current sampling rate to approximately $43 \mathrm{kHz}$, as three channels are sampled. Furthermore, since four current samples (two of each current) are required for estimation, the applied voltage pulsewidth should be at least about $38 \mu \mathrm{s}$ (corresponds to $26 \mathrm{kHz}$ ) in order for this to be possible. This means that, at low speeds, the estimates during the applied voltage periods often cannot be used, as the voltage pulses are narrow. Consequently, fewer position estimates are available for further filtering, which together with the increased effect of A/D quantization and noise, should create poorer estimates at low speeds. This conjecture is confirmed by the experimental results presented in Section VI.

The fact that the sampling is not simultaneous imposes some difficulties in processing the current samples for use in the estimation algorithm. To compensate for delay in sampling the $b$-phase current relative to current in $a$ phase, the first sample of $i_{b_{m}}$ at the beginning of the considered switching interval is linearly extrapolated one sampling interval backward as the slope of the current ripple is known. The resultant value is then used as $i_{b_{m}}$ in (5) and has been experimentally shown to improve the estimator accuracy. 


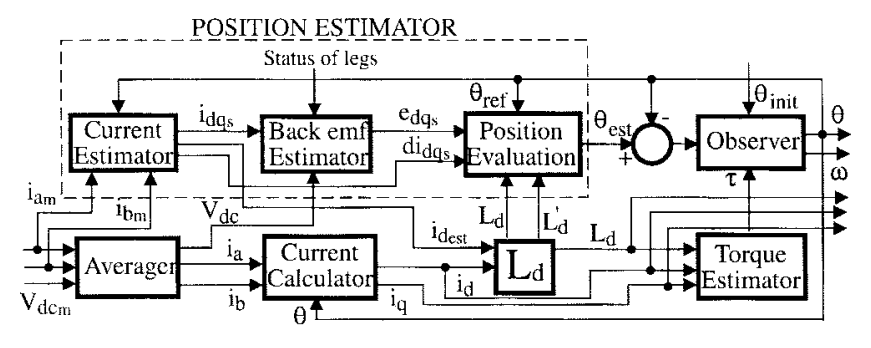

Fig. 2. Sensorless algorithm.

\section{Sensorless Control Algorithm}

The heart of the sensorless control algorithm shown in Fig. 2 is the position estimator based on the solutions to (9). The output from this algorithm is fed into a closed-loop observer based on the load model equations of (1) [10]. The merit of using an observer is that both angular velocity $\omega$ and position $\theta$ can be accurately predicted without any knowledge of past information and, hence, without delay, which is crucial for high-performance control. The convergence of the control algorithm and machine operating stability are simply a matter of appropriately tuning the observer gains, the main criteria being the quality of the estimates being produced by the position estimation algorithm. If the estimates are known to be good, then the observer feedback gain is increased, otherwise, it is decreased. Clearly, this implies that gain scheduling is required to get good estimates throughout the entire speed range of the machine.

The position estimator evaluates the electrical angle of the $d$ axis for each of the legs switching instants of the previous control interval using current samples $i_{a_{m}}$ and $i_{b_{m}}$ and average dc-link voltage $V_{\mathrm{dc}}$. It then selects the best estimate $\theta_{\mathrm{est}}$, i.e., the one having the least variation from the observer last prediction $\theta_{\text {ref. }}$. The $\theta_{\text {ref }}$ is also used to estimate the $d$-axis currents $i_{d_{\text {cst }}}$ for indexing the lookup tables containing the $L_{d}$ and $L_{d}^{\prime}$ values required for calculating $\tilde{L}_{d}$ and then the position estimates. The average $d q$ currents $\left(i_{d}\right.$ and $\left.i_{q}\right)$ and the corresponding $d$-axis inductances are obtained similarly, but using the average phase currents $\left(i_{a}\right.$ and $\left.i_{b}\right)$.

The $\theta_{\text {est }}$ value and the average torque $\tau$ estimated using (1) are fed as an input to the observer to generate a filtered $\theta$ and $\omega$ relevant at the beginning of the next control interval. The $\theta$ and $\omega$ estimates, as well as $i_{d}$ and $i_{q}$, are then used throughout the rest of the vector control algorithm for the control prediction. How this is achieved is discussed in Section IV.

\section{A. Startup Procedure}

In order to properly initialize the observer (which is necessary for its initial position estimate to be accurate), the rotor initial position $\theta_{\text {init }}$ should be found as accurately as possible. A simple startup procedure is carried out for this purpose. It utilizes a minimum reluctance principle, i.e., the tendency of the rotor $d$ axis to align with the stator MMF phasor. The idea is, therefore, to set up the average current vector $\bar{i}$ in a desired angle $\varphi$ with respect to the $a$ axis (Fig. 1) and then allow the rotor to take up the alignment position when it is obvious that $\theta=\theta_{\text {init }} \approx \varphi$. Clearly, this simple procedure will not work in every situation. For example, the initial $\bar{i}$ could coincide with

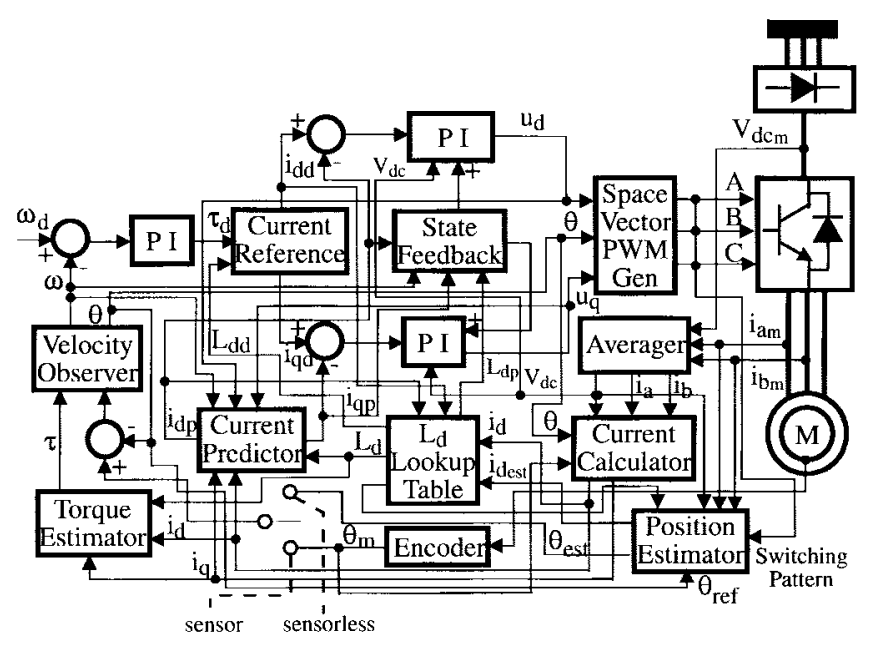

Fig. 3. Experimental vector controller.

the $q$ axis, and no alignment torque would result. This potential problem can be easily overcome by specifying two different spatial angles for $\bar{i}$, such that $\left|\varphi_{2}-\varphi_{1}\right| \approx 45^{\circ}$. The previous condition is introduced just to have a reasonably high starting torque while moving the rotor from its initial $d$-axis (or $q$-axis) alignment position $\varphi_{1}$ to a new one $\left(\theta_{\text {init }} \approx \varphi_{2}\right)$. In order to improve the test accuracy, the current magnitude should be large enough to provide the torque sufficient to surpass bearing friction around the alignment position.

The real-time implementation includes a digital current proportional integral (PI) controller with circular voltage limiting identical to that used in the vector controller. The difference is that it performs its actions in the stationary $d_{s}-q_{s}$ frame (Fig. 1). The $d_{s} q_{s}$ components of the desired current phasor $\underline{i}$ are used as references to the current loops, whereas the feedback $d_{s} q_{s}$ currents are obtained from the average phase currents $i_{a}$ and $i_{b}$ using (5). The desired output voltages from the controller are then passed into a PWM algorithm.

\section{EXPERIMENTAl CONTROLler Design}

A functional block diagram of the algorithmic structure of the Syncrel speed vector controller is shown in Fig. 3. The current loops (one for each rotor axis) and angular velocity loop are all conventional PI regulators with integrator antiwindup [11]. An obvious advantage of the controller is a possibility of controlling the machine speed with or without a shaft position sensor. However, only the issues related to the sensorless control are of interest in this paper.

The control algorithm and the startup procedure, including the PWM waveform generation, are implemented entirely in software written in $\mathrm{C}$ using floating-point arithmetic. This was a design decision to allow the maximum flexibility in the experimental system. The control computer is a $90-\mathrm{MHz}$ Intel Pentium-processor-based PC. An advantage of this platform is its low cost and the large variety of software development tools available. System parameters are monitored with the integrated PC screen display. The inverter hardware and encoder are interfaced to the CPU bus using two programmable I/O boards with interrupt capabilities, the control signal generation board 
and $\mathrm{A} / \mathrm{D}$ data conversion board. These are considered in more detail in Section V.

The control code itself essentially consists of the main program and two interrupt routines servicing the boards interrupts.

\section{A. Main Program}

The main program carries out any operator interfacing. Before running the main loop, it initializes the software, programs up the I/O boards and Direct Memory Access (DMA) controller, generates contactors closing signals, and sets up interrupts.

The main loop polls the keyboard to check for the program stop command or the new values for the speed reference, as well as current magnitude and angle while running the initial rotor position startup procedure. The user can also select and change on-line the desired control variables to be displayed on the PC monitor or oscilloscope. Similarly, the transition to execution of the actual control algorithm (after determination of the initial rotor position) is accomplished by a user command.

Another important function of the program main loop is examining the status of the hardware trip indication bit of the control board port. Upon normal termination of the main loop (stop signal received or hardware trip detected), the program does the usual post-interrupt procedure, resets the hardware, and opens all the contactors.

\section{B. A/D Board Interrupt Routine}

The "DMA complete" interrupt routine is fairly straightforward. It sets up the A/D board for the next set of $A / D$ conversions and then converts the contents of the DMA buffer into floating-point numbers representing the actual measurements. These correspond to $i_{a_{m}}, i_{b_{m}}$ and $V_{\mathrm{dc}_{\mathrm{m}}}$ in Figs. 2 and 3. It is the task of the user to ensure that the samples are available at the beginning of each control interval. The maximum number of samples that allows this is 19 per channel, i.e., 57 in total.

The use of DMA means that the processor is not burdened down with handling relatively high-frequency interrupts from the A/D card. It was found that removing these interrupts resulted in a significant decrease in the execution time of the control algorithm.

\section{Control Interrupt Routine}

The control interrupt routine first runs the startup procedure and then the maximum torque per ampere control algorithm in Fig. 3. The control rate of $2 \mathrm{kHz}$ provides enough time for both the DMA interrupt and the control interrupt routines to be executed before the next control interrupt occurs. The first priority of the routine is to predict the control to be applied to the machine in the next control interval and to output this to the inverter hardware. The control flow required to achieve this objective is as follows.

- A/D conversions and DMA circuitry are triggered by sending out the appropriate bit pattern to the A/D board control register.
- Samples corresponding to the previous control interval are averaged and either the startup procedure or the sensorless algorithm from Fig. 2 is run.

- A simple regeneration control strategy is implemented. The associated bit on the control board output port is pulsed high to switch the regeneration transistor on whenever the average dc-link voltage exceeds the userspecified value. When the voltage drops below the lower limit, the bit is pulsed low and device is turned off.

- The control $d q$ currents $i_{d p}$ and $i_{q p}$ are predicted using a simple Euler approximation of the current differentials in (2). This and the remainder of the algorithm up to the PWM waveform generation are disabled while carrying out the startup procedure.

- The speed PI algorithm is executed to generate the desired torque $\tau_{d}$. The current reference generator then determines the minimum currents $i_{d d}$ and $i_{q d}$ required for the machine to produce this torque. The optimal $i_{d d}$ versus $\tau_{d}$ characteristic is precomputed using Matlab and stored in a lookup table.

- The predicted state feedback $d q$ voltages are calculated to compensate for the rotational voltages. These appear to the current regulators as unknown state disturbances [12].

- The current PI controllers are run to obtain the predictions for the $d q$ voltages to be applied to the machine. These are fed into the space-vector-based PWM generator [9] together with $\theta$. In the case of the startup procedure, the $\theta$ value is zero, as the current controller is then stator oriented.

- The desired $d q$ voltages are converted into the corresponding inverter legs switching pattern by executing the PWM algorithm with overlap (dead) time compensation [13].

- The switching times are programmed into the appropriate timers to generate the actual firing waveforms for the insulated gate bipolar transistors (IGBT's) in the next control interval.

- The function which programs D/A converters (DAC's) on the control board (Fig. 4) is called if the user wants some variables to be viewed on the oscilloscope.

\section{PC INTERFACE BOARDS}

\section{A. A/D Board}

The 12-b A/D board is a base model of the commercial Data Translation DT2821 series [14]. Samples of analogue measurement signals from the system are transferred to a PC memory using DMA. The memory segments used for the DMA buffer are programmed into the 8237 DMA controller and are initialized prior to DMA/data transfer, since the controller is programmed in autoinitialization mode. This is important, as it saves code execution time, because the controller has to be initialized only once at the beginning of the program. The detailed description of the steps for programming both the board and DMA controller can be found in [14]. 


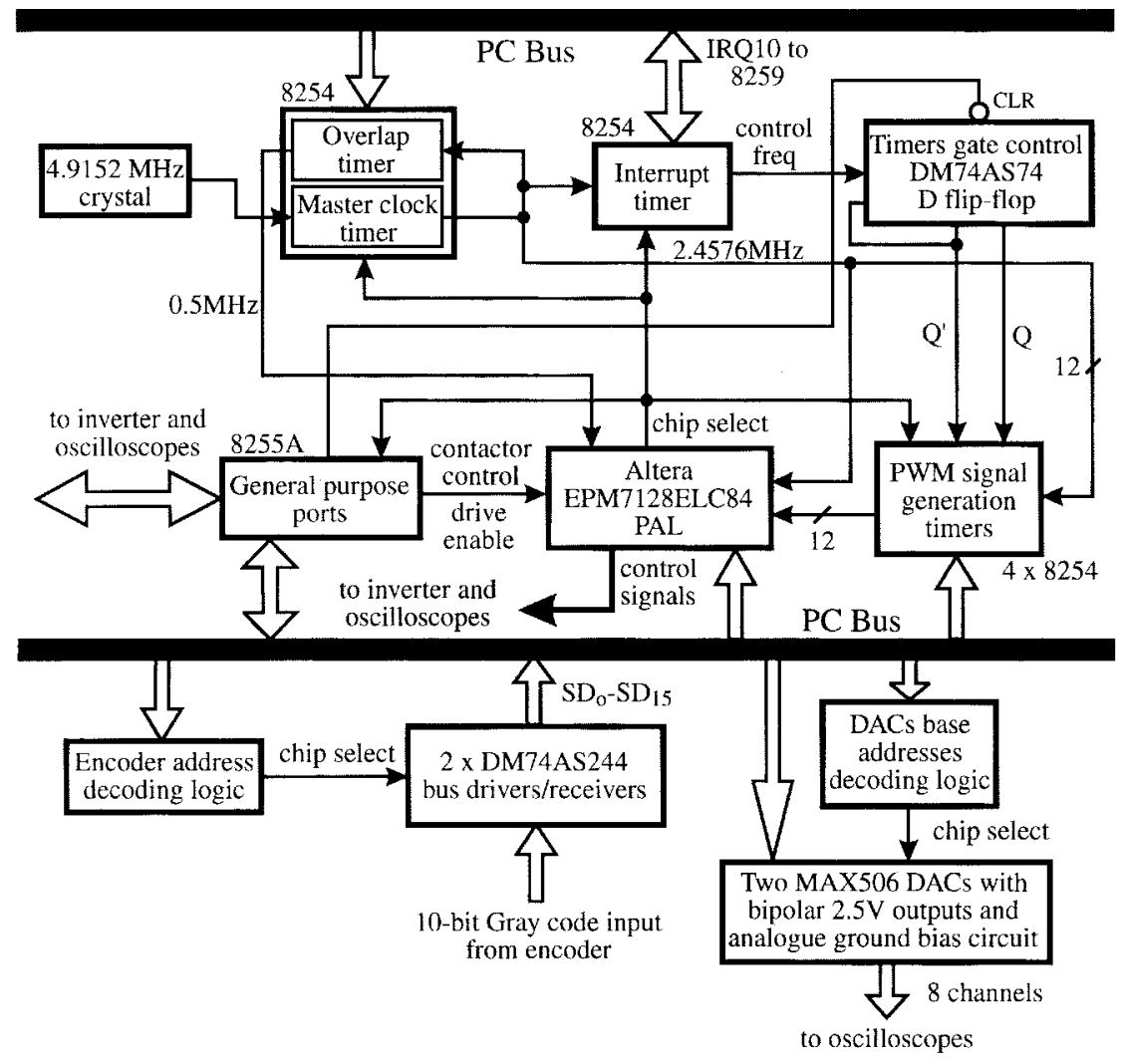

Fig. 4. Control signal generation board.

\section{B. Control Board}

The simplified functional block diagram of the control board is presented in Fig. 4. Its major function is to generate the control signals for firing the inverter transistors and closing and opening contactors. This is essentially achieved using five lots of 8254 interval timers and a single $8255 \mathrm{~A}$ chip with three 8-b ports.

The master timer is clocked from a local 4.9152-MHz quartz crystal oscillator, which produces a master clock signal. It is programmed in a square-wave mode with the minimum count (2) for the highest possible clock rate $(2.4576 \mathrm{MHz})$ and, hence, the best resolution.

The interrupt timer operates in the same mode and generates an interrupt request to the 8259 controller at the $2-\mathrm{kHz}$ control rate. Its output signals are also fed to a toggle flip-flop, which controls the gates of the PWM waveform generation timers.

The overlap timer is also programmed to operate in a continuous square-wave mode. It produces a clock signal which is used by a programmable array logic device (PAL) for the generation of the nonoverlapping firing waveforms for the IGBT's. The period of this signal (which is user programmable) is the dead time between the top and bottom devices of the same inverter leg being turned off/on. This is to prevent the possibility of shoot through.

The drive signal generation timers are programmed in software-triggered strobe mode. They are used in pairs to produce the desired three-phase firing waveforms. Each pair is associated with a single transistor of the inverter (except for the regeneration). One timer is used to switch the top device on and the bottom off, and the other is vice versa. The presence of two timers allows their gate inputs to be connected to complementary outputs from a toggling flip-flop, which provides a low signal into the appropriate timer upon a control interrupt. This design ensures that there is no timing skew in the generation of the PWM.

The coordination circuit for all the actions carried out by the control board is a PAL. It performs the address decoding, accepts and processes the output signals from the timers and ports, and generates the resultant control signals, which are then transferred to the inverter hardware. The PWM waveform signals are also available for viewing on an oscilloscope.

Another interface to the inverter and oscilloscopes is a $8255 \mathrm{~A}$ programmed in the basic $\mathrm{I} / \mathrm{O}$ mode. The input port bits are used to monitor the state of the inverter power devices and hardware trip condition and can be read at any time. Output ports bits are dedicated to opening/ closing contactors, drive enable signals, regeneration transistor control, gate control flip-flop clear signal, and an oscilloscope trigger.

The bottom part of Fig. 4 is a block diagram of the interface system for the encoder and oscilloscopes to the PC bus. The encoder subsystem connects the 10-b Gray code measurements to the 16-b data bus by means of two octal buffers with common enable gates. The translation of the Gray code input into its binary equivalent is done in software using a 1024-word-long lookup table (due to noise problems with a PAL-based translator).

The DAC subsystem consists of eight 8-b DAC's that enable monitoring of several software variables on an oscilloscope. 

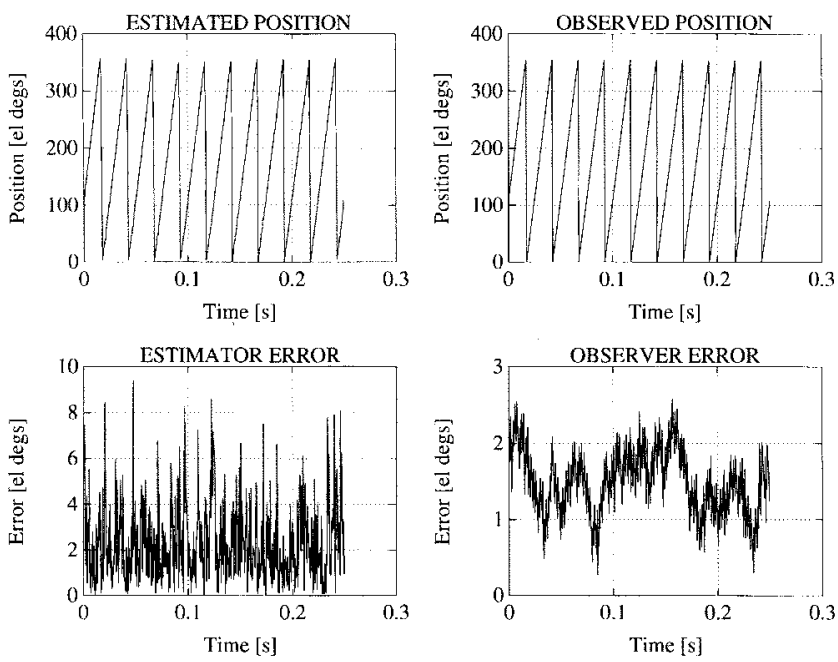

Fig. 5. Rotor position estimates and estimation errors at $1200 \mathrm{r} / \mathrm{min}$.

\section{EXPERIMENTAL RESULTS}

Experimental results have been generated for a $5.8-\mathrm{kW}$ axially laminated Syncrel (see the Appendix) executing maximum torque per ampere sensorless control, as shown in Fig. 3. The machine was running unloaded at various speeds down to zero with full voltage on the dc link $(\approx 600 \mathrm{~V})$.

The plots in Fig. 5 present the estimated electrical position of the rotor obtained directly from (10) $\left(\theta_{\mathrm{est}}\right)$, the position after passing $\theta_{\text {est }}$ through an observer $(\theta)$, and the absolute variations of these estimates from 10-b absolute encoder measurements. The machine speed was $1200 \mathrm{r} / \mathrm{min}$. The raw estimates, $\theta_{\text {est }}$, are fairly accurate (average error is $2.4^{\circ}$ ), despite error spikes which have been found to be mainly due to the noise effects, measurement quantization, and sensitivity to parameter knowledge inaccuracies. A significant improvement in accuracy is achieved by processing $\theta_{\text {est }}$ through the observer. The average error is reduced to approximately $1.5^{\circ}$, with the maximum values being about $2.5^{\circ}$ or less.

In order to demonstrate the validity and very high accuracy of the sensorless algorithm at very low speeds, the machine was driven at $100 \mathrm{r} / \mathrm{min}$. The plots for this case are shown in Fig. 6. The estimator performance, as predicted, deteriorates with decreasing the speed. The $\theta_{\text {est }}$ estimates are much noisier than at $1200 \mathrm{r} / \mathrm{min}$, the error ripples being occasionally larger than $50^{\circ}$. The average estimation error is, however, still reasonably low $\left(7.4^{\circ}\right)$. The effectiveness of the observer as a low-pass filter is more than obvious from the same figure. The accuracy of the filtered noisy $\theta_{\text {est }}(\theta)$ used in the control procedure is substantially better. The peak error is about $3.5^{\circ}$ or less, with the average being $1.8^{\circ}$, only marginally worse than the high-speed case.

Fig. 7 clearly illustrates the good controller performance under both the transient and steady-state conditions of the machine. The top plot is the Syncrel response to a varying speed reference between $\pm 100 \mathrm{r} / \mathrm{min}$. The speed reversal is affected with very little overshoot. The bottom figure shows the machine speed while changing the reference values between $\pm 200 \mathrm{r} / \mathrm{min}$ and zero. It can be seen that the speed can be effectively and stably controlled, even at standstill. In
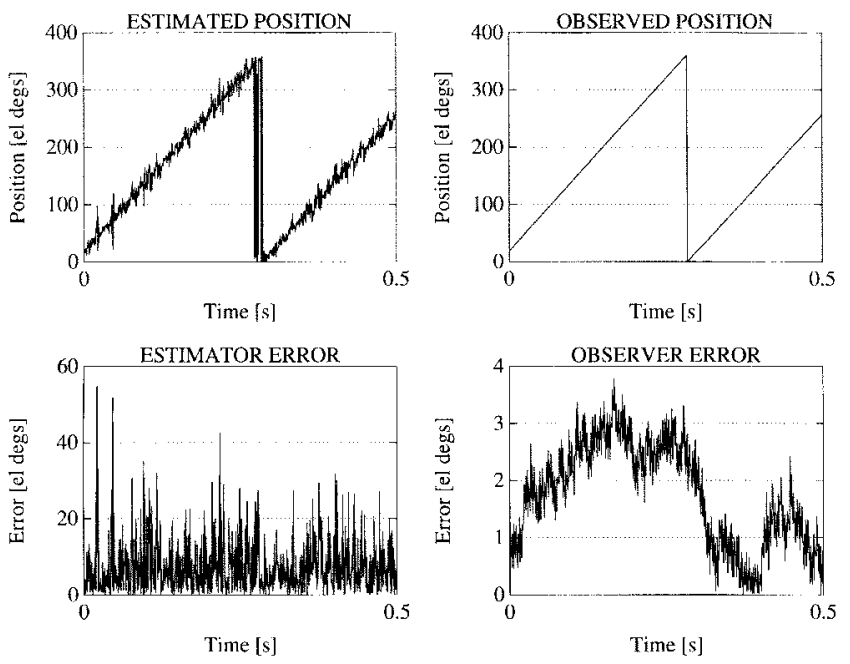

Fig. 6. Performance of position estimator and observer at $100 \mathrm{r} / \mathrm{min}$.
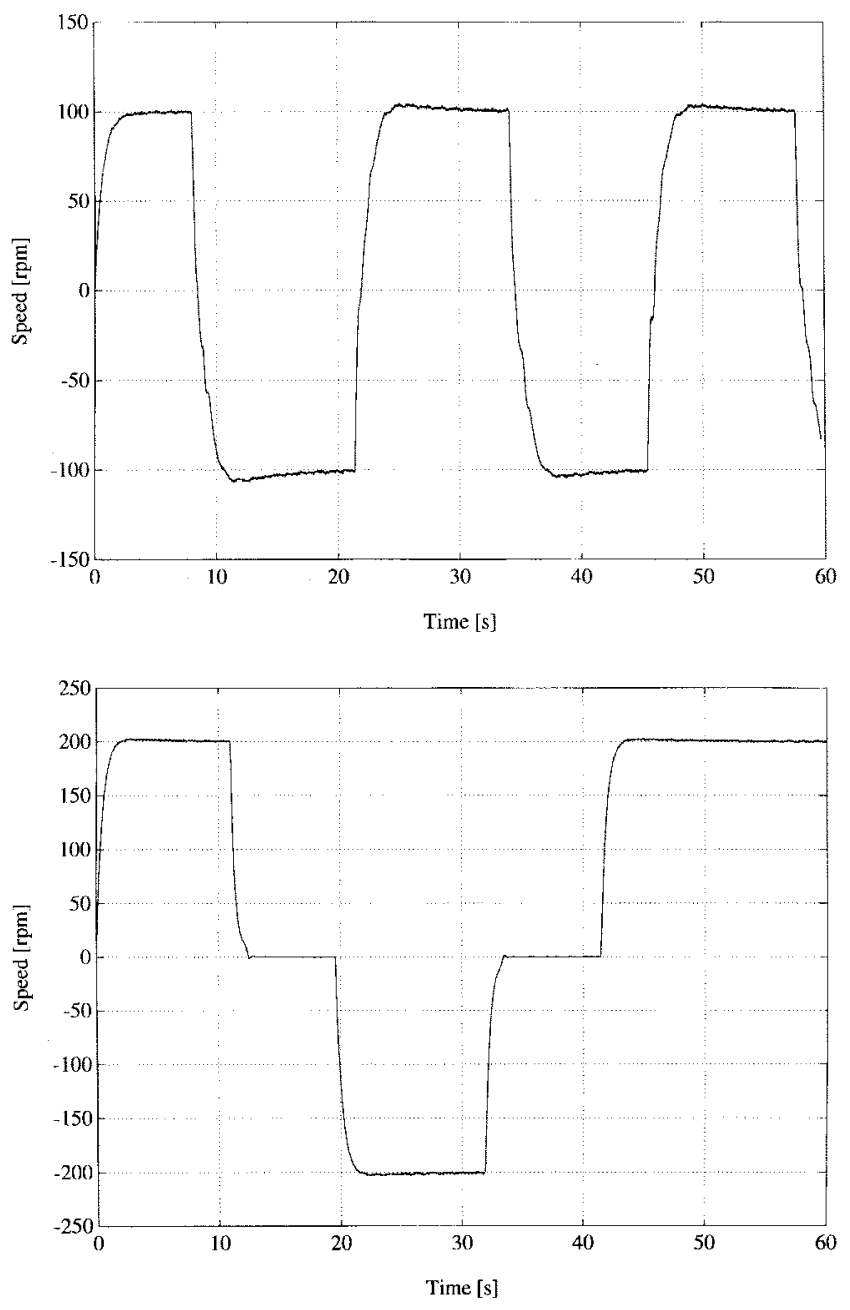

Fig. 7. Sensorless controller performance at low speeds.

this case, and for low-speed machine operation in general, the gains of both the speed PI regulator and the observer must be lowered, as instability and divergency of the control algorithm may be experienced due to noisy input estimates. This results in low bandwidth control and relatively slow dynamic response 
of the machine. It is important to note, however, that both speed characteristics are very smooth and accurately follow the desired trajectories. The similar curves can be naturally obtained for higher speeds when the estimation accuracy is better.

\section{CONCLUSIONS}

The main contribution of this paper is the development of a new, effective sensorless control algorithm that implements the maximum torque per ampere strategy for the Syncrel. The high performance of the sensorless vector controller is confirmed by experimental results for a $5.8-\mathrm{kW}$ axially laminated machine. The advantages of the presented sensorless algorithm over the existing ones discussed in the introduction can be summarized as follows.

- The algorithm is applicable over the entire speed range of the machine, including standstill.

- The rotor position is estimated on-line at the control rate, allowing the controller to effectively replace the encoder instantaneous measurements, with obvious implications on high quality control.

- The algorithm does not require the injection of any special signals or special inverter switching techniques. Instead, it works using the current ripple that is inherent with PWM voltage control.

- The very high instantaneous accuracy of both the position and angular velocity estimates at either speed is achieved using a conventional load model-based observer. The electrical position estimation error was shown to be less than $2^{\circ}$ electrical.

One limitation is a requirement for a simple startup procedure to determine rotor initial position before executing the control algorithm. This is yet to be investigated, as the authors believe that it can be eliminated by relying on the position estimation technique itself. A further limitation is the parameter dependence inherent with this technique. Online parameter estimation techniques are being investigated. The fulfillment of these two objectives would further improve viability of the control algorithm.

\section{APPENDIX \\ EXPERIMENTAL MACHINE}

The test machine is an inverter-fed Syncrel having a commercial DF132M frame size, $7.5-\mathrm{kW}$ three-phase Y-connected induction machine stator, and an axially laminated rotor. The IGBT-based inverter and the rotor were both designed and built at the workshop of the Department of Electrical and Computer Engineering, University of Newcastle. The rotor was constructed based on a design from the University of Glasgow [15].

The 10-kW inverter uses a Mitsubishi CM50TF-24E sixtransistor power module and a CM50E3Y-24E device from the same manufacturer for regeneration. Both component sets are rated at $1200 \mathrm{~V}$ and $50 \mathrm{~A}$, with the switching frequency up to $20 \mathrm{kHz}$. Hall-effect transducers and precise potential dividers were used to measure machine currents and inverter
TABLE I

SYNCREL PARAMETERS

\begin{tabular}{c||c}
\hline \hline Power & $5.8-\mathrm{kW}$ \\
Voltage (rms) & $415-\mathrm{V}$ \\
Current (rms) & $14.3-\mathrm{A}$ \\
Current angle & $60^{\circ}$ \\
Speed & $1500-\mathrm{rpm}$ \\
Poles & 4 \\
\hline \hline$L_{d} / L_{q}$ unsaturated & 9.1 \\
$L_{d} / L_{q}$ saturated & 7.8 \\
$L_{q}$ & $9.8-\mathrm{mH}$ \\
$R_{d c}$ (cold) & $0.6-\Omega$ \\
$J$ (machine-load) & $0.23-\mathrm{kgm}{ }^{2}$ \\
\hline \hline Stator slots & 36 \\
Air-gap & $0.48-\mathrm{mm}$ \\
Rotor diameter & $126-\mathrm{mm}$ \\
Rotor length & $175-\mathrm{mm}$ \\
Insulator thickness & $0.38-\mathrm{mm}$ \\
Lamination thickness & $0.35-\mathrm{mmm}$ \\
Lamination-insulation layers & 43 \\
Pole arc & $120^{\circ} \mathrm{clcc}$ \\
Pole picce & brass \\
\hline \hline
\end{tabular}

dc-link voltage. Torque was measured using a $100-\mathrm{N} \cdot \mathrm{m}$ torque transducer.

The load was a $30-\mathrm{kW}$ dc machine with a through shaft enabling the attachment of a 10-b absolute encoder. It was fed from a Ward-Leonard-based dc supply, which allowed simple regeneration back into the three-phase mains.

The Syncrel maximum torque performance and design parameters are summarized in Table I. As the rotor is not optimally designed, the machine is capable of developing only about $5.8 \mathrm{~kW}$ at the rated speed and current. One of the major causes for this relatively modest power production is the small airgap $(0.48 \mathrm{~mm}$ as compared to $0.517 \mathrm{~mm}$ in [15]). Furthermore, difficulties in getting $0.5-\mathrm{mm}$ grainoriented steel laminations in Australia have imposed the use of standard transformer laminations, which has resulted in less steel being present in the rotor. Consequently, it has lower iron losses, but saturates easier, compromising a saliency ratio and performance of the Syncrel prototype.

The total inertia constant $(J)$ was determined by conducting a simple step-torque test, the speed response of the unloaded Syncrel being monitored on a digital oscilloscope. The linearized load model equation in (1) was then used to predict $J$ from a knowledge of the rate of change of angular velocity (neglecting friction).

The inductances were measured by running an instantaneous flux-linkage locked rotor test [16]. The results obtained can be accurately represented by the following sixth-order polynomial:

$$
\begin{aligned}
L_{d}= & 1.489 \times 10^{-5} i_{d}^{6}-1.2865 \times 10^{-3} i_{d}^{5}+4.3288 \times 10^{-2} i_{d}^{4} \\
& -0.70486 i_{d}^{3}+5.5906 i_{d}^{2}-21.565 i_{d}+121.77[\mathrm{mH}]
\end{aligned}
$$

where $i_{d} \geqslant 4.9 \mathrm{~A}$. For $i_{d} 94.9 \mathrm{~A}, L_{d}$ is naturally unsaturated.

\section{ACKNOWLEDGMENT}

The authors would like to acknowledge T. Wylie, who constructed much of the experimental system hardware, and P. McLauchlan and R. Hicks, who built the Syncrel rotor. 


\section{REFERENCES}

[1] M. Schroedl and P. Weinmeier, "Sensorless control of reluctance machines at arbitrary operating conditions including standstill," IEEE Trans. Power Electron., vol. 9, pp. 225-231, Mar. 1994.

[2] R. Lagerquist, I. Boldea, and T. J. E. Miller, "Sensorless control of the synchronous reluctance motor," IEEE Trans. Ind. Applicat., vol. 30, pp. 673-682, May/June 1994.

[3] M. S. Arefeen, M. Ehsani, and T. A. Lipo, "An analysis of the accuracy of indirect shaft sensor for synchronous reluctance motor," IEEE Trans. Ind. Applicat., vol. 30, pp. 1202-1209, Sept./Oct. 1994.

[4] M. Jovanovic, R. E. Betz, and D. Platt, "Position and speed estimation of sensorless synchronous reluctance motor," in Proc. IEEE PEDS'95 Conf., Singapore, Feb. 1995, pp. 844-849.

[5] Y. Q. Xiang and S. A. Nasar, "Estimation of rotor position and speed of a synchronous reluctance motor for servodrives," Proc. Inst. Elect. Eng., vol. 142, no. 3, pp. 201-205, May 1995.

[6] T. Matsuo and T. A. Lipo, "Rotor position detection scheme for synchronous reluctance motor based on current measurements," IEEE Trans. Ind. Applicat., vol. 31, pp. 860-868, July/Aug. 1995.

[7] Z. I. Boldea and S. A. Nasar, "Torque vector control (tvc) of axiallylaminated anisotropic (ala) rotor reluctance synchronous motors," Elect. Mach. Power Syst., vol. 19, pp. 381-398, 1991.

[8] P. L. Jansen and R. D. Lorenz, "Transducerless position and velocity estimation in induction and salient ac machines," IEEE Trans. Ind. Applicat., vol. 31, pp. 240-247, Mar./Apr. 1995.

[9] H. W. VanDerBroeck, H. C. Skudelny, and G. V. Stanke, "Analysis and realization of a pulsewidth modulator based on voltage space vectors," IEEE Trans. Ind. Applicat., vol. 24, pp. 142-150, Jan./Feb. 1988.

[10] R. D. Lorenz and K. W. VanPatten, "High-resolution velocity estimation for all-digital, ac servo drives," IEEE Trans. Ind. Applicat., vol. 27, pp. 701-705, July/Aug. 1991.

[11] K. J. Astrom and B. Wittenmark, Computer-Controlled Systems. Englewood Cliffs, NJ: Prentice-Hall, 1990.

[12] R. D. Lorenz and D. B. Lawson, "Performance of feedforward current regulators for field-oriented induction machine controllers," IEEE Trans. Ind. Applicat., vol. 23, pp. 597-602, July/Aug. 1987.

[13] R. B. Sepe and J. H. Lang, "Inverter nonlinearities and discrete-time vector current control," IEEE Trans. Ind. Applicat., vol. 30, pp. 62-70, Jan./Feb. 1994.

[14] User Manual for DT2821 Series, Data Translation, Marlboro, MA, 1987.

[15] W. L. Soong, D. A. Staton, and T. J. E. Miller, "Design of a new axially-laminated interior permanent magnet motor," IEEE Trans. Ind. Applicat., vol. 31, pp. 358-367, Mar./Apr. 1995.

[16] C. Cossar and T. J. E. Miller, "Electromagnetic testing of switched reluctance motors," in Proc. ICEM, 1992, pp. 470-474.

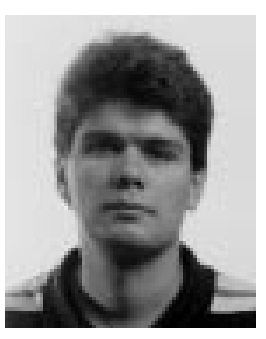

Milutin G. Jovanović received the Dipl.Eng. and M.E.E. degrees from the University of Belgrade, Belgrade, Yugoslavia, in 1987 and 1991, respectively, and the Ph.D. degree from the University of Newcastle, Newcastle, Australia, in 1997.

He is currently a Lecturer in the School of Electrical Engineering, Electronics and Physics, Liverpool John Moores University, Liverpool, U.K. His major interests lie in the areas of electrical machines and drives, industrial electronics, and power systems.

Dr. Jovanović is a member of the Industrial Drives Committee of the IEEE Industry Applications Society.

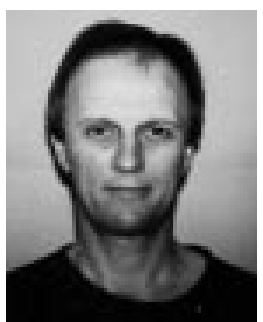

Robert E. Betz (M'92) received the B.E., M.E., and Ph.D. degrees from the University of Newcastle, Newcastle, Australia in 1979, 1982, and 1984, respectively.

He is currently a Senior Lecturer in the Department of Electrical and Computer Engineering, University of Newcastle. His major interests are electrical machine drives, real-time operating systems, and industrial electronics.

Dr. Betz is a member of the Industrial Drives Committee of the IEEE Industry Applications Society.

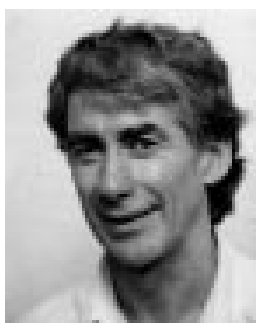

Don Platt (S'86-M'87) received the B.Sc. and B.E. degrees in electrical engineering from the University of New South Wales, Sydney, Australia, in 1970 and the Ph.D. degree from the University of Wollongong, Wollongong, Australia, in 1988.

$\mathrm{He}$ has worked in several industries, including both light and heavy processing industries. He is presently a Senior Lecturer in the Department of Electrical and Computer Engineering, University of Wollongong, Wollongong, Australia. His areas of interest include magnetic circuits, electric machines and drives, and power electronics. 